\title{
MOSAIC-MAPPING OF VERY EXTENDED OBJECTS IN (SUB)MILLIMETRE AND NEAR-INFRARED
}

\author{
SABINE PHILIPP AND ROBERT ZYLKA \\ Max-Planck-Institut für Radioastronomie \\ Auf dem Hügel 69, 53121 Bonn, Germany \\ Institut für Theoretische Astrophysik \\ Tiergartenstrasse 15, 69121 Heidelberg, Germany
}

The details of current and past star formation episodes and the stellar content of the central parsecs of our galaxy are still not very well known (Mezger et al., 1996). We have therefore begun an observing program to study star formation processes in the nuclear bulge. Here we discuss the technical aspects of this program, while the scientific goals are presented by Zylka et al. (1997).

To survey of the central $1.2^{\circ} \times 0.25^{\circ}$ in the $\mathrm{mm} / \mathrm{submm}$ continuum, we used the MPIfR bolometer arrays at the IRAM $30-\mathrm{m}$ telescope. While scanning continuously in alternating directions, data were acquired during very short integrations (on-the-fly scan mapping, OTF). To eliminate the influence of the atmosphere, we switched between two positions while scanning (double-beam mapping). The remaining atmospheric noise is still much higher than the receiver noise. This correlated noise can be very sucessfully filtered out from data obtained with array receivers (Fig. 1).

The NIR mapping of the central $0.5^{\circ} \times 0.25^{\circ}$ was performed with the IRAC2B camera at the ESO/MPG 2.2m-telescope. The "sky" reference was chosen in the direction of a local very dark cloud, roughly $13.5^{\prime}$ away from Sgr A*. This fixed "sky" position plays an important role in ensuring that we obtain the same zero level intensity for the whole data set.

In principle, the combination of several OTF maps or images to one mosaic requires the same algorithms. The reduction of the $\mathrm{mm} / \mathrm{submm}$ and the NIR data was performed with the same software package MOPSI ${ }^{1}$, developed by R. Zylka. The main difference between datasets obtained with radio/(sub)mm-telescopes and optical telescope is the ratio of the effective resolution and the pointing accuracy. The pointing-accuracy of

${ }^{1} M$ ap $O$ n-Off $P$ ointing $S$ kydip Image 
radio/(sub)mm-telescopes is clearly better than their resolution, while for the optical/NIR-telescopes this is usually not the case. Automatic correction of the coordinates in the latter case is therefore necessary (Fig. 2).

\section{References}

Mezger P.G., Duschl W.J., Zylka R., 1996, A\&AR, 7, 4.

Zylka R. et al., 1997, in these proceedings.
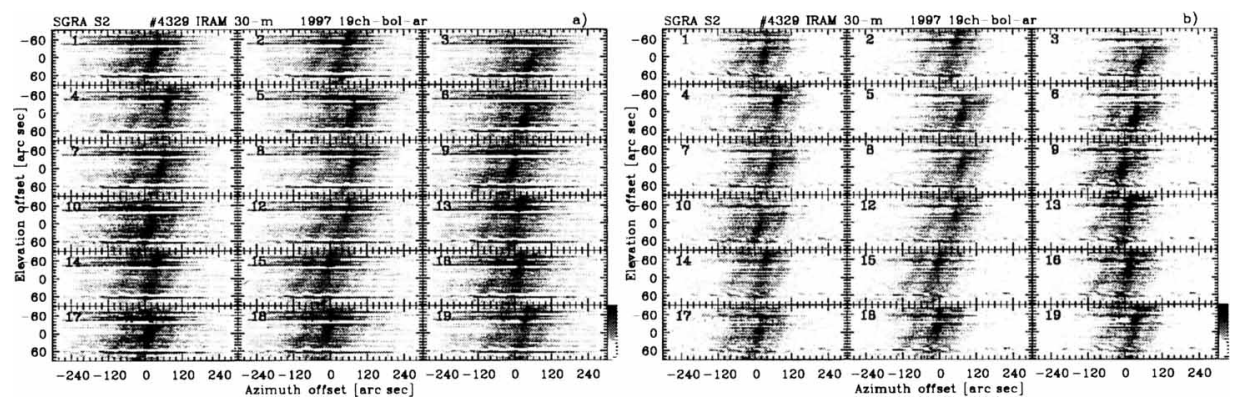

Figure 1. Map of the $20 \mathrm{~km} / \mathrm{s}$-cloud (M-0.13-0.08), south of the Galactic Centre, obtained with the 19-channel bolometer array detector in February 1997 during moderate weather-conditions. Bolometer \#11 showed much higher noise, therefore was not used in the analysis. Figure a: Data reduced without the sky noise filter. Figure b: The same data after filtering out of the atmospheric noise with the algorithm developed by R. Zylka.

K Band Mosaic

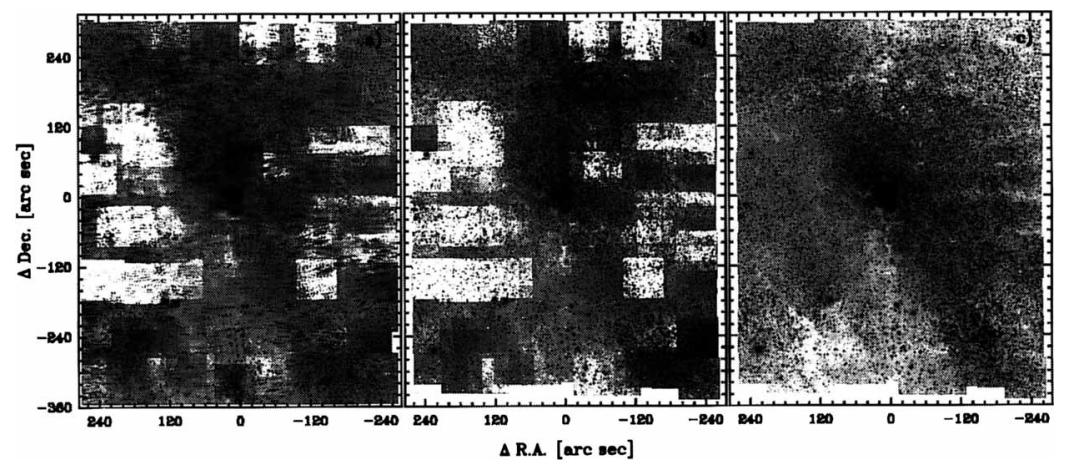

Figure 2. Data reduction steps and about $20 \%$ of the final mosaic in K. The data were acquired in June and July 1996. The mosaic shown consists of about 300 images, while the final mosaic will contain about 2000 images. Figure a) shows the mosaic combined from the non-calibrated, unaligned images; mosaic b) the same data after coordinate correction ("alignment"); and c) the properly calibrated K band mosaic. The images are plotted on a logarithmic intensity scale. The coordinates are offsets from the position of Sgr A*. 